\title{
Effects of pattern complexity in solving pattern recognition and pattern production problems
}

\author{
GARY A. DAVIS AND ALICE J. TRAIN \\ WISCONSIN RESEARCH AND DEVELOPMENT CENTER FOR COGNITIVE LEARNING
}

Using a switch-light problem-solving apparatus, the task of 27 college Ss was either to identify each light pattern or to learn to produce each pattern. Incrcasing the number of lights in the pattern increased the difficulty of both tasks. Participation in the pattern recognition task appeared to facilitate performance in the production task. Performance in the production task was not facilitated by informing $S$ of which pattern was to be produced.

In a pattern production switch-light problemsolving task, $S$ is required to manipulate switches on his response panel until he achieves a particular pattern of lights (Davis, 1967; Davis, Train, \& Manske, in press). The switch-light apparatus also can be used for pattern recognition problems, in which S's task is to identify which of several light patterns has been preprogrammed into the apparatus. According to a study of pattern recognition strategies by Peterson \& Hillner (1967), S will usually approach a pattern recognition task by pressing every switch just once, then selecting the correct pattern from the population of patterns provided by E. Thus one finding of Peterson and Hillner was that light patterns defined by three, four, or five lights did not differ in difficulty as measured by switch presses to criterion.

The purpose of the present experiment was to investigate the effects of number of lights, hence number of required switches, upon performance in pattern recognition vs pattern production tasks.

\section{Method}

The stimulus display unit consisted of a 3 by 4 matrix of 1-in. lights placed 4 in. apart upon a black relay rack panel. The response panel consisted of a 3 by 4 matrix of spring-return lever switches mounted on a standard chassis. The response unit, fixed to the open type relay rack just below and in front of the stimulus display, sloped toward S. When pressed, each switch connected to a light would change the state of that light from off to on or on to off. A switchboard-like arrangement on the back of the relay rack allowed $\mathrm{E}$ to connect any switch to any light. Further apparatus details are available in Davis (1967).

Three 3-light patterns ( $L$, triangle, line), three 4-light patterns (check, square, diamond), and three 5-light patterns ( $X$, cross, inverted $T$ ) were used. The three, four, or five reluvant switches used for a given problem were randomly selected from the pool of 12 switches. Across all nine problems, every switch was relevant approximately equally often.
Each of the nine Ss in Group RP (RecognitionProduction) first learned to identify, or recognize, which pattern was programmed into the machine. He was instructed to press switches until he could indicate which one of the 15 patterns on a prepared diagram (nine actually used, six dummy) was correct. After identifying the pattern, S's task was then to learn which switches would produce that pattern. He pressed switches until the complete light pattern appeared. Then E turned the lights off, and the procedure was repeated until $S$ could select the three, four, or five relevant switches without error. He then proceeded to another recognition task with a new pattern.

In Group IP (Informed-Production) and Group P (Production), the nine Ss in each group solved only the production problems, i.e., they repeatedly produced each pattern until they could select the correct switches without error. The only difference between these two groups was that the Ss in Group IP were shown which pattern they were to produce before each problem, while Ss in Group $P$ were not.

All Ss in Groups RP, IP, and P solved all nine problems. Problem order was determined by the rows of a 9 by 9 Latin square. The main dependent variable for both tasks was total switch presses to criterion.

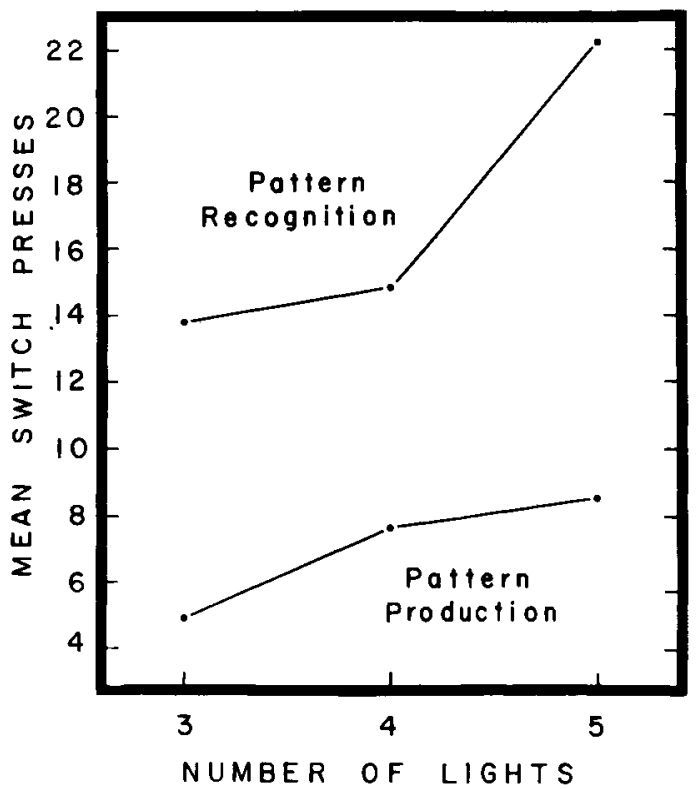

Fig. 1. Mean switch presses to criterion in the pattern recognition and pattern production tasks of Group RP. 


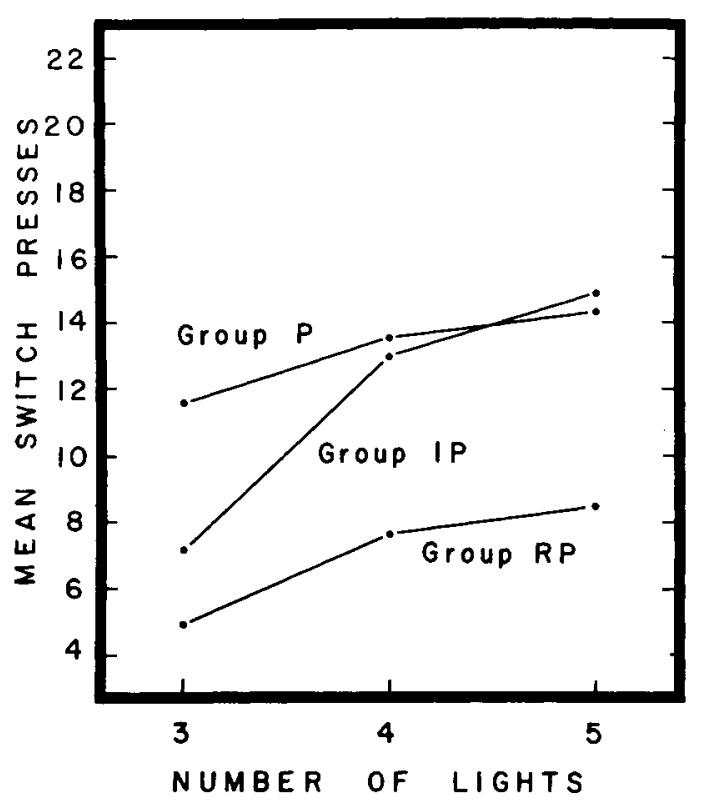

Fig. 2. Mean switch presses to criterion in the pattern production task of Groups RP, IP, and P.

\section{Results}

Considering only the pattern recognition vs pattern production tasks of Group RP, Fig. 1 shows that increasing the number of required lights led to a fairly systematic increase in switch presses to criterion for both tasks. An analysis of variance on the combined data from both tasks showed the effect of number of lights to be quite reliable ( $F=7.60$, df $=2 / 118, p<.01$ ) with no task by number of lights interaction $(F<1.0)$. In an analysis of variance on pattern recognition scores alone, the effect of number of lights was again significant $(F=4.98, d f=2 / 56$, $\mathrm{p}<.025)$. Subsequent tests showed the 5-1ight mean to be significantly larger than the 4-1ight mean ( $t$ $=6.95, \mathrm{df}=56, p<.01)^{2}$ and the 3-light mean $(t=7.88$, $\mathrm{df}=56, p<.01)$. The difference between the 3-light mean and the 4-1ight mean was not significant. In neither analysis did variance attributable to specific patterns, beyond the variance due to number of lights, approach significance.

Figure 2 shows the pattern production switch press scores for Groups RP, IP, and P. The effect of number of lights was significant $(F=4.23, d f=2 / 180, p$ $<.025)$. Subsequent tests showed that the 5-light mean and the 4-light mean did not significantly differ, while the 5-light mean was significantly larger than the 3-light mean $(t=2.80, d f=180, p<.01)$ and the 4-light mean was significantly larger than the 3-light mean $(t=2.09, d f=180, p<.05)$. Variance attributable to specific pattern differences, beyond the variance due to the number of lights, was not significant $(F<1.0)$.
Regarding group differences, two trends are noticeable. First, performance in Group RP appears to have been facilitated by participating in the prior pattern recognition task. However, the F-ratio for the groups main effect did not reach an acceptable level of significance $(F=2.75, d f=2 / 24, p<.10)$. Second, the mean switch press scores of Groups IP and $\mathbf{P}$ were essentially identical, although there was a slight difference favoring Group IP with the easier 3-light problem.

\section{Discussion}

Contrary to the results of the Peterson \& Hillner (1967) study, increasing the number of lights (or number of required switches) led to more trialand-error switch-pressing behavior in the pattern recognition task as well as in the pattern production task. In regard to pattern recognition strategies, many Ss in the 5-light problems pressed each switch exactly twice before identifying the pattern, rather than sampling all of the switches just once as they typically did with the 3- and 4-light problems. In the pattern production task, as in earlier studies (Davis, 1967; Davis, Train, \& Manske, in press), increasing the number of required switches systematically impaired performance, due in part to the additional trial-and-error behavior and in part to the additional memory requirements with the longer response chain.

A comparison of pattern production performance by Groups RP, IP, and P suggests that participation in the pattern recognition task facilitated performance in the following production task (Group RP), indicating that during the recognition task Ss incidentally or deliberately learned the particular switches which controlled the light pattern. Of the 81 pattern production problems solved by Ss in Group RP, 16 were in fact solved on the first trial without error. Regarding Groups IP and P, there was apparently no uniform facilitation by telling the $S$ in advance exactly which pattern he was to learn to produce.

\section{References}

DAVIS, G. A. Detrimental effects of distraction, additional response alternatives, and longer response chains in solving switch-light problems. J. exp. Psychol., 1967, 73, 45-55.

DAVIS, G. A., TRAIN, A. J., \& MANSKE, M. E. Trial and error vs. "insightful" problem solving: Effects of distraction, additional response alternatives, and longer response chains. $J$. exp. Psychol., in press.

PETERSON, M. J., \& HILLNER, S. D. Learning to solve pattern identification problems. J. exp. Psychol., 1967, 73, 328-390.

Notes

1. This research was performed pursuant to contract $\mathrm{OE}$ 5-10-154 with the United States Office of Education, Department of Health, Education, and Welfare, under the provisions of the Cooperative Research Program.

2. All tests are two-tailed. 